\title{
Fotoluminescência do InGaAs/InP Crescido pela Técnica de Epitaxia por Feixe Molecular
}

\author{
Photoluminescence of InGaAs/InP Grown \\ by Molecular Beam Epitaxy
}

\author{
L. C. Poças ${ }^{1}$, E. M. Lopes ${ }^{1}$, J. L. Duarte ${ }^{1}$, I. F. L. Dias ${ }^{1}$, E. Laureto ${ }^{1}$, D. O. T. \\ Filho ${ }^{1}$, P. S. S. Guimarães ${ }^{2}$, L. A. Cury ${ }^{2}$, J. C. Harmand ${ }^{3}$.
}

\section{Resumo}

Medidas de fotoluminescência (PL) em função da temperatura e da potência de excitação foram realizadas em uma amostra contendo uma camada de $\mathrm{In}_{0.53} \mathrm{Ga}_{0,47}$ As crescida pela técnica de epitaxia por feixe molecular-MBE (Molecular Beam Epitaxy) sobre um substrato de InP. As origens dos vários processos de luminescência observados à baixa temperatura foram determinados estudando seus diferentes comportamentos com o aumento da temperatura e da potência de excitação e comparando os resultados com os dados encontrados na literatura. Foram identificadas: uma transição envolvendo éxcitons localizados e duas transições envolvendo impurezas aceitadoras. Uma revisão dos principais trabalhos publicados na literatura relacionados às transições ópticas observadas à baixa temperatura no InGaAs/ InP também é apresentada.

Palavras chaves: InGaAs/InP. Fotoluminescência.

\begin{abstract}
Photoluminescence (PL) measurements due to temperature and excitation power were carried out in as function of sample containing a In0,53Ga0,47. As layer, grown by Molecular Beam Epitaxy on an InP substrate. The origins of the several luminescence processes observed at low temperature were determined by studying their different behaviors with increasing temperature and excitation power and by comparing the results with the data found in the literature. The following transitions have been identified: one transition involving localized excitons and two transitions involving acceptor impurities. A review of the main works published in the literature related to the optical transitions observed at low temperature in $\mathrm{InGaAs} / \mathrm{InP}$ is also presented.

Key words: InGaAs/InP. Photoluminescence.
\end{abstract}

1 Departamento de Física - CCE - UEL - Londrina/PR.

2 Departamento de Física - ICEx - UFMG, Belo Horizonte/MG.

3 Laboratoire de Photonique et de Nanostructures, CNRS, Marcoussis, France. 


\section{Introdução}

Sistemas InGaAs/InP são particularmente atrativos, devido a sua aplicação em optoeletrônica e em dispositivos eletrônicos de alta velocidade (PEARSALL, 1993), sendo materiais básicos em vários dispositivos tais como: fotodetetores (SENGUPTA et al., 1997; WEISS et al., 2000), guia de ondas (WEISS et al., 2000; LEWÉN et al., 2002; DORREN et al., 2000), moduladores (WEISS et al., 2000; DORREN et al., 2000; GEDDO; BELLANI; GUIZZETTI, 1994), conversores termofotovoltáicos (TPV) (GINIGE et al., 2004), "lasers" de grande comprimento de onda (WEISS et al., 2000; BASSIGNANA; MINER; PUETZ, 1989; LOUATI et al., 1987) e "lasers" de cascata quântica (QCL) (TROCCOLI et al., 2003). Em particular, a energia de "bandgap" em torno de $0,75 \mathrm{eV}$, à temperatura ambiente, torna o InGaAs/InP estratégico em sistemas de comunicação operando entre 1,1 e 1,7 $\mu \mathrm{m}$ (CHEN; KIM, 1981), região que abrange a janela de baixa dispersão $(1,3 \mu \mathrm{m})$ e a de mínima atenuação $(1,5 \mu \mathrm{m})$ das fibras ópticas (MORRAL et al., 2003).

$\mathrm{O}$ conhecimento preciso de parâmetros importantes do sistema $\operatorname{In}_{1-\mathrm{x}} \mathrm{Ga}_{\mathrm{x}} \mathrm{As} / \mathrm{InP}$, especialmente a energia de "bandgap" para a composição de rede casada $(x=0,468)$, é fundamental para $\mathrm{o}$ aprimoramento de sua aplicação em dispositivos. Apesar disso, existe na literatura uma grande dispersão com relação aos valores atribuídos à energia de "bandgap" do InGaAs crescido em rede casada com o InP (de 801 a $822 \mathrm{meV}$ em baixas temperaturas e de 725 a $749 \mathrm{meV}$ em $\mathrm{T}=300 \mathrm{~K}$ ). Uma explicação para tal dispersão deve-se ao fato que, em grande parte destes estudos, a presença de tensões devidas às diferenças na composição da liga com relação à composição de rede casada é ou desprezada ou não explorada (GASKILL et al., 1990), e a grande maioria dos trabalhos considera $x$ $=0,47$ como a concentração de rede casada, ao invés de $x=0,468$. Sabe-se que, no caso do $\operatorname{In}_{1-x} \mathrm{Ga}_{x} \mathrm{As} /$ InP, pequenos desvios da concentração de rede casada é suficiente para provocar mudanças significativas na sua estrutura de bandas o que, por sua vez, induz a uma variação na energia de "bandgap" em relação ao sistema sem tensão (GEDDO; BELLANI, GUIZZETTI, 1994). Dessa forma, a condição de casamento de rede é um importante parâmetro a ser cuidadosamente controlado durante o crescimento do semicondutor, para que se obtenham estruturas de boa qualidade.

Neste trabalho, nós investigamos as propriedades ópticas do sistema InGaAs/InP utilizando a técnica de fotoluminescência $(\mathrm{PL})$. Nós realizamos medidas de PL em função da temperatura e da intensidade de excitação, com objetivo de determinar a energia de "bandgap" para o InGaAs/InP. Nós identificamos os diversos processos de luminescência observados à baixa temperatura $(8 \mathrm{~K})$, por meio da análise de seus comportamentos, com aumento da temperatura e da intensidade de excitação e pela comparação com os dados encontrados na literatura.

\section{Propriedades Ópticas do InGaAs/InP - revisão da literatura}

Existe na literatura uma grande dispersão com relação aos valores atribuídos ao "bandgap" ou para a energia da transição excitônica no InGaAs crescido em rede casada com o InP, dos quais apresentamos os resultados que consideramos mais representativos:

- Gaskill et al. (1990) citam a dispersão na literatura $(0,801$ a $0,822 \mathrm{eV})$, e obtêm o valor de $\mathrm{E}_{\mathrm{g}}=0,803$ $\mathrm{eV}$ para o $\mathrm{In}_{0,53} \mathrm{Ga}_{0,47}$ As a $0 \mathrm{~K}$, extrapolando dados obtidos de medidas de fotorefletância.

- Radhakrishnam et al. (2000) utilizam $\mathrm{E}_{\mathrm{g}}=0,801$ $\mathrm{eV}$ para o $\mathrm{In}_{0,53} \mathrm{Ga}_{0,47} \mathrm{As}$.

- Bassignana, Miner e Puetz (1989) obtêm para a transição excitônica do $\mathrm{In}_{0.53} \mathrm{Ga}_{0.47}$ As o valor de 0,801 $\mathrm{eV}$, deduzido de medidas de PL a $7 \mathrm{~K}$.

- Goetz et al. (1983) obtiveram o valor de $\mathrm{E}_{\mathrm{g}}=0,811$ $\mathrm{eV}$ para o $\mathrm{In}_{0,532} \mathrm{Ga}_{0,468} \mathrm{As}$ a $2 \mathrm{~K}$ e determinaram a energia de ligação do éxciton em 2,1 $\pm 0,1 \mathrm{meV}$. Esses valores foram deduzidos de medidas de raio-X, PL e absorção.

- Hybertsen (1991) utilizam o valor de $\mathrm{E}_{\mathrm{g}}=0,810$ $\mathrm{eV}$ para o $\mathrm{In}_{0,53} \mathrm{Ga}_{0,47} \mathrm{As}$.

- Böhrer, Krost e Bimberg (1993) observaram $\mathrm{E}_{\mathrm{g}}=$ $0,811 \mathrm{eV}$ para o $\mathrm{In}_{0,53} \mathrm{Ga}_{0,47}$ As à temperatura de $2 \mathrm{~K}$ pela técnica de PL. 
- Towe (1982), por meio de medidas de PL em função da temperatura, extrapolam o valor de $\mathrm{E}_{\mathrm{g}}=0,821 \mathrm{eV}$ para o $\mathrm{In}_{0,53} \mathrm{Ga}_{0,47}$ As a $0 \mathrm{~K}$.

- Lugand et al.(1997), utilizando a técnica de PL, observaram a transição excitônica em 0,790 eV para o In ${ }_{0,53} \mathrm{Ga}_{0,47}$ As à temperatura de $4 \mathrm{~K}$.

- Pearsall; Eaves e Portal (1983), utilizando a técnica de PL, observaram uma transição excitônica em torno de $0,810 \mathrm{eV}$ à temperatura de $2 \mathrm{~K}$ em uma amostra relativamente pura de $\mathrm{In}_{0,53} \mathrm{Ga}_{0,47} \mathrm{As}$.

- Louati et al. (1987) e Yu e Kuphal (1984), usando a dependência teórica de Varshni, obtiveram para a posição do pico de PL do $\mathrm{In}_{0,53} \mathrm{Ga}_{0,47} \mathrm{As}$, respectivamente: $\mathrm{E}_{\mathrm{g} 0}=0,810 \mathrm{eV}$ e $\mathrm{E}_{\mathrm{g} 0}=0,814 \mathrm{eV}$.

- Fry et al.(1985) observaram o pico de éxciton ligado em 0,810 eV para o InGaAs (rede casada com o InP) em medidas de PL realizadas a 4,2 K.

Acreditamos que os valores mais precisos sejam os obtidos por Bassignana, Miner e Puetz (1989) (transição excitônica em 0,801 eV ) e Gaskill et al. (1990), ( $\mathrm{E}_{\mathrm{g}}=$ $0,803 \mathrm{eV}$ a $0 \mathrm{~K}$ ), pois esses dois trabalhos levam em consideração os efeitos da variação na composição e a tensão associada sobre a energia de "bandgap". Além disso, no último trabalho, o valor de $\mathrm{E}_{\mathrm{g}}$ foi obtido de medidas de fotorefletância e, embora a energia de "bandgap" possa ser estimada através de espectros de PL, é mais apropriado o uso de uma técnica associada ao processo de absorção óptica, que é intrínseco à técnica de fotorefletância. No entanto, valores de $\mathrm{E}_{\mathrm{g}}$ em torno de $0,810-0,813 \mathrm{eV}$ para o $\mathrm{In}_{0,53} \mathrm{Ga}_{0,47} \mathrm{As}$ têm sido recentemente utilizados (DAÍ; FAN; CHEN, 1998) e citados na literatura científica como sendo os valores comumente aceitos (YANG; CHEN; LI, 2000).

Dependendo de características específicas da amostra como concentração de impurezas e das condições experimentais como temperatura e intensidade de excitação, são vários os mecanismos de recombinação envolvendo o éxciton que podem ser observados em uma medida de PL, como por exemplo: éxcitons livres $(\mathrm{X})$, éxcitons ligados à impureza doadora $\left(\mathrm{D}^{\circ}, \mathrm{X}\right)$ e éxciton ligado à impureza aceitadora $\left(\mathrm{A}^{\mathrm{o}}, \mathrm{X}\right)$. Esses mecanismos se caracterizam por linhas de emissão cujas energias são localizadas um pouco abaixo da energia de "bandgap" do semicondutor.
Goetz et al. (1983) usaram o modelo hidrogênico para determinar as energias de ligação do éxciton e do doador, $\mathrm{E}_{\mathrm{x}}$ e $\mathrm{E}_{\mathrm{D}}$ respectivamente, e utilizaram para os coeficientes $\alpha$ e $\beta$ (definidos na $2^{\mathrm{a}}$ coluna da Tabela I) o valor de $\sim$, 1 determinado pela regra de Haynes, e, assim, calcularam a separação em energia de algumas linhas excitônicas em relação ao "bandgap" fundamental do $\operatorname{In}_{0,53} \mathrm{Ga}_{0,47} \mathrm{As}$. Alguns destes resultados são apresentados na Tabela I.

Tabela I. Posição espectral das diversas linhas excitônicas calculadas por Goetz et al. (1983). E $\mathrm{g}_{\mathrm{g}}$ a energia do "bandgap" fundamental, $\mathrm{E}_{\mathrm{x}}$ é a energia de ligação do éxciton livre e $\mathrm{E}_{\mathrm{D}}$ e $\mathrm{E}_{\mathrm{A}}$ representam, respectivamente, as energias de ligação das impurezas doadoras e aceitadoras.

\begin{tabular}{c|c|c}
\hline $\begin{array}{c}\text { Processo de } \\
\text { recombinação }\end{array}$ & $\begin{array}{c}\text { Energia de emissão teórica em } \\
\text { termos dos coeficientes } \alpha \text { e } \beta\end{array}$ & $\begin{array}{c}\text { Separação de " } \mathrm{E}_{\mathrm{g}} \text { " } \\
\text { utilizando } \alpha \text { e } \beta \cong 0,1 \\
(\mathrm{meV})\end{array}$ \\
\hline$(\mathrm{X})$ & $\mathrm{E}_{\mathrm{g}}-\mathrm{E}_{\mathrm{X}}$ & 2,0 \\
\hline$\left(\mathrm{D}^{\circ}, \mathrm{X}\right)$ & $\mathrm{E}_{\mathrm{g}}-\left(\mathrm{E}_{\mathrm{X}}+\alpha \mathrm{E}_{\mathrm{D}}\right)$ & 2,3 \\
\hline$\left(\mathrm{A}^{\circ}, \mathrm{X}\right)$ & $\mathrm{E}_{\mathrm{g}}-\left(\mathrm{E}_{\mathrm{X}}+\beta \mathrm{E}_{\mathrm{A}}\right)$ & $3,3 \ldots 4,5$ \\
\hline
\end{tabular}

$\mathrm{Na}$ Tabela II, mostramos os valores correspondentes à separação em energia entre os picos de emissão envolvendo impurezas e o pico de emissão excitônica encontrados na literatura, e na tabela III, as energias de ionização das impurezas em relação ao "bandgap" do InGaAs.

Tabela II. Separação em energia $(\Delta \mathrm{E})$ entre o pico de PL de impurezas aceitadoras e o pico de PL excitônico no $\mathrm{In}_{0,53} \mathrm{Ga}_{0,47}$ As (BIMBERG; SROCKA, 1993).

\begin{tabular}{c|c|c|c}
\hline $\begin{array}{c}\text { Impureza } \\
\text { aceitadora }\end{array}$ & $\begin{array}{c}\Delta \text { E em relação a } \\
\text { transição excitônica } \\
(\mathrm{meV})\end{array}$ & $\mathrm{T} \mathrm{(K)}$ & Ref. \\
\hline $\mathrm{C}$ & $8 \pm 1$ & 2,4 & $1,2,3$ \\
\hline $\mathrm{Mg}$ & 13 & 4 & 4 \\
\hline $\mathrm{Zn}$ & $17 \pm 1$ & 2 & 1 \\
& 19 & 4 & 2 \\
$\mathrm{Si}$ & $26 \pm 1$ & 4 & 3 \\
\hline $\mathrm{Hg}$ & $30 \pm 1$ & 4 & 1 \\
\hline $\mathrm{Be}$ & $62 \pm 5$ & 4 & 4 \\
\hline
\end{tabular}

${ }^{1}$ (GOETZ et al., 1983); ${ }^{2}$ (FRY et al., 1985); ${ }^{3}$ (BHATTACHARYA;RAO; TSAI, 1983); ${ }^{4}$ (LOUATI et al., 1987); ${ }^{5}$ (FENG et al., 1979). 
Tabela III. Energia de ionização de impurezas aceitadoras em relação ao "bandgap" do $\operatorname{In}_{0,53} \mathrm{Ga}_{0,47}$ As (BIMBERG; SROCKA, 1993).

\begin{tabular}{c|c|c}
\hline $\begin{array}{c}\text { Impureza } \\
\text { aceitadora }\end{array}$ & $\begin{array}{c}\text { Energia de ionização em } \\
\text { relação ao "bandgap”. } \\
(\mathrm{meV})\end{array}$ & Ref. \\
\hline $\mathrm{C}$ & $13 \pm 1$ & $1,2,3$ \\
\hline $\mathrm{Mg}$ & $16 \pm 2$ & 4 \\
\hline $\mathrm{Cd}$ & 19 & 6 \\
& $23,4 . .6,1$ & 1,2 \\
\hline $\mathrm{Zn}$ & $22 \pm 1$ & 3 \\
\hline $\mathrm{Be}$ & $28 \pm 1$ & 5 \\
\hline $\mathrm{Si}$ & $\sim 27$ & 1 \\
\hline $\mathrm{Hg}$ & $25 \pm 1$ & 4 \\
\hline
\end{tabular}

1 (GOETZ et al., 1983);' (FRY et al., 1985); (BHATTACHARYA; RAO; TSAI, 1983); ${ }^{4}$ (LOUATI et al., 1987); ${ }^{5}$ (FENG et al., 1979); ${ }^{6}$ (CHEN et al., 1989); ${ }^{7}$ (WEHMANN; FIELDLER; SCHLACHETZKI, 1986).

De acordo com a literatura (BIMBERG; SROCKA, 1993), as transições envolvendo impurezas mais comumente observadas por PL no InGaAs (além da emissão excitônica a que já nos referimos) são as emissões da banda de condução para impureza aceitadora (e-A) e a recombinação envolvendo o par doador-aceitador (D-A) (GOETZ et al., 1983; BÖHRER; KROST; KIMBERG, 1993; PEARSALL, 1983; CHARREAUX;GUILLOT; NOUAILHAT, 1986; BHATTACHARYA; RAO; TSAI, 1983; YU; PENG; MORKOC, 1989; SWAMINATHAN et al., 1985).

Pearsall, Eaves e Portal (1983) observaram para o $\mathrm{Ga}_{0,47} \mathrm{In}_{0.53}$ As uma transição D-A posicionada em $\mathrm{Eg}-0,019 \mathrm{eV}$, com a participação do Zn como impureza aceitadora.

Dhar et al. (1997) investigaram amostras de $\mathrm{Ga}_{0,47} \mathrm{In}_{0,53} \mathrm{As}$ :Er e observaram picos de emissão que foram atribuídos ao $\mathrm{Zn}$ aceitador com energia de ionização de $\sim 21 \mathrm{meV}$ e ao Si aceitador com energia de ionização de $\sim 35 \mathrm{meV}$.
Charreaux, Guillot e Nouailhat (1986) identificaram por meio de ajustes teóricos das bandas D-A observadas em amostras $\mathrm{Ga}_{0,47} \mathrm{In}_{0,53} \mathrm{As}$ a presença das impurezas aceitadoras $\mathrm{Zn}, \mathrm{C}$, e Si, cujas energias de ionização são, respectivamente, $22 \pm 1$, $15 \pm 1$ e $25 \pm 1$.

Yu, Peng e Morkoc (1989), usando medidas de $\mathrm{PL}$, investigaram camadas de $\mathrm{Ga}_{\mathrm{x}} \operatorname{In}_{1-\mathrm{x}}$ As $(0,44 \leq \chi \leq$ $0,48)$ crescidas por MBE sobre substratos de InP e observaram transições D-A, com a participação do $\mathrm{C}$ como impureza aceitadora. Eles relataram uma dispersão na literatura para a energia de ionização atribuída ao C entre 13 e $19 \mathrm{meV}$ e obtiveram, para suas amostras, uma energia de ionização de $\sim 17 \mathrm{meV}$.

\section{Detalhes Experimentais}

Na Figura 1, apresentamos um esquema com a sequiência de crescimento das camadas da amostra SR295. Essa amostra contém duas super-redes não dopadas crescidas uma logo acima da outra: a mais interna (SR1) é constituída de 20 poços quânticos de InGaAs de $100 \AA$ e barreiras de InAlAs de $50 \AA$, e a mais externa (SR2), de 20 poços quânticos de InGaAs de $80 \AA$ e barreiras de InAlAs de $100 \AA$. As camadas foram crescidas pela técnica de epitaxia por feixe molecular (MBE) sobre um substrato de InP. Entre o substrato e a SR1 foi crescida uma camada "buffer" de InGaAs com 0,25 $\mu \mathrm{m}$ de espessura, que tem como objetivo acomodar as camadas subseqüentes e impedir a difusão de impurezas do substrato para dentro dos poços quânticos.

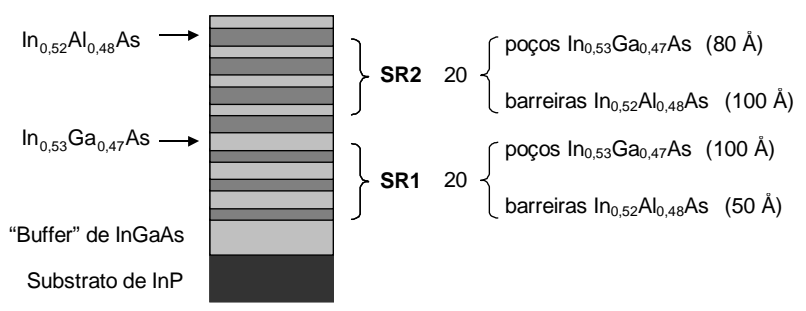

Figura 1. Esquema de crescimento epitaxial das camadas da amostra SR295. 
As medidas de PL foram realizadas usando como fonte de excitação, a linha $5145 \AA$ de um "laser" de $\mathrm{Ar}^{+}$com diâmetro de "spot" de $300 \mu \mathrm{m}$. A análise espectral da luminescência foi realizada utilizando um monocromador com distância focal de $0,50 \mathrm{~m} \mathrm{e}$ a detecção foi feita por meio de um fotodiodo pin de InGaAs refrigerado termoeletricamente, utilizando o procedimento padrão de amplificação "lock-in".

\section{Resultados Experimentais}

Na Figura 2, apresentamos um espectro de PL obtido à temperatura de $8 \mathrm{~K}$ para a amostra SR295. Neste espectro é possível observar em uma região de maior energia, dois picos, posicionados em 0,865 e $0,890 \mathrm{eV}$, que têm sua origem em recombinações devidas às duas super-redes existentes na amostra (SR1 e SR2). Na inserção, salientamos uma banda relativamente larga entre 0,756 e $0,800 \mathrm{eV}$ que pela posição em energia deve ter sua origem na camada "buffer" de InGaAs existente na amostra. É importante notar que a emissão na região espectral das super-redes é extremamente mais intensa do que a emissão da camada "buffer" de InGaAs. Vale lembrar que a intensidade de excitação que atinge as super-redes (especialmente a mais externa SR2) certamente é muito maior do que a intensidade de excitação que chega até a camada "buffer".

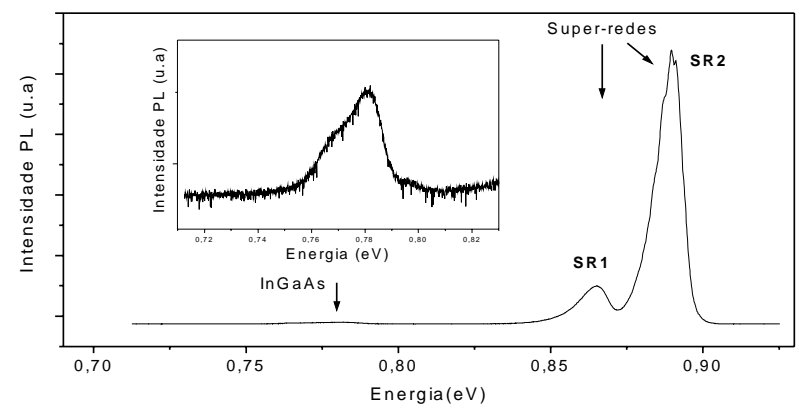

Figura 2. Espectro de fotoluminescência da amostra SR295 obtido a $8 \mathrm{~K}$.

$\mathrm{Na}$ sequiência deste trabalho, analisaremos as mudanças provocadas nos espectros de PL obtidos na região de emissão da camada "buffer" de InGaAs (que passaremos a nos referir como região do InGaAs), quando submetidas à variação de temperatura e potência. Buscamos, dessa forma, identificar os processos envolvidos nesta banda de emissão.

Na Figura 3, mostramos um ajuste da forma de linha, onde utilizamos o programa Origin 5.0 professional, em um espectro obtido à temperatura de $8 \mathrm{~K}$ na região do InGaAs, onde verificamos a existência de pelo menos três canais de recombinação presentes em nossa amostra a baixas temperaturas.

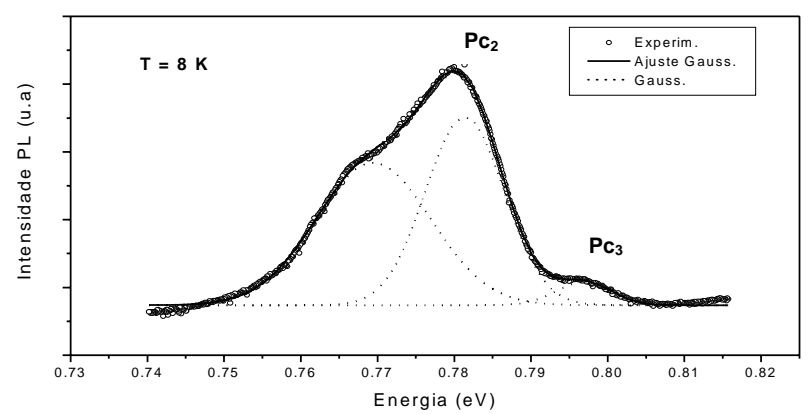

Figura 3. Ajuste por três gaussianas para o espectro da amostra SR295 obtido à temperatura de 8K.

Passaremos a nos referir aos três canais de recombinação presentes nos espectros da amostra SR295, de acordo com sua posição em energia, como: $\mathbf{P c}_{1}$ - o pico de menor energia, $\mathbf{P c}_{\mathbf{2}}$ - o pico de energia intermediária e $\mathbf{P c}_{\mathbf{3}}$ - o pico de maior energia. Os valores obtidos por meio do ajuste para a energia, largura de linha e a área dos picos da Fig. 3, são mostrados na Tabela IV.

Tabela IV. Valores obtidos pelo ajuste por três gaussianas mostradas na Fig. 3, para o espectro de PL à temperatura de $8 \mathrm{~K}$.

\begin{tabular}{c|c|c|c}
\hline Transição & $\begin{array}{c}\text { Energia } \\
(\mathrm{eV})\end{array}$ & $\begin{array}{c}\text { Largura de linha à meia } \\
\text { altura }(\mathrm{meV})\end{array}$ & $\begin{array}{c}\text { Área sob a curva } \\
(\mathrm{meV})\end{array}$ \\
\hline $\mathbf{P c}_{\mathbf{1}}$ & 0,772 & 17,7 & 3,17 \\
\hline $\mathbf{P c}_{\mathbf{2}}$ & 0,782 & 9,3 & 2,1 \\
\hline $\mathbf{P c}_{3}$ & 0,797 & 8,1 & 0,24 \\
\hline
\end{tabular}


Nas próximas sub-secções, tentaremos determinar a origem desses três canais de recombinação, primeiramente analisando as posições dos picos de emissão, à baixa temperatura, e depois, pela análise do comportamento desses picos com a variação de temperatura e da potência de excitação.

\section{Análise Pela Posição em Energia}

Pico de maior energia $\mathrm{Pc}_{3}$

Da Tabela IV, verificamos que o pico de maior energia $\mathbf{P c}_{3}$ obtido por meio do ajuste está posicionado em $0,797 \mathrm{eV}$, à temperatura de $8 \mathrm{~K}$. De acordo com Bimberg e Srocka (1993), a análise da dependência dos espectros de PL do InGaAs, em função da intensidade de excitação e da temperatura, revela que os picos próximos do "bandgap" são dominados por éxcitons ligados, o que tem sido verificado em diversos trabalhos publicados (BASSIGNANA; MINER; PUETZ, 1989; GOETZ et al., 1983; BÖHRER; KROST; BIMBERG, 1993; CHARREAUX; GUILLOT; NOUAILHAT, 1986; BHATTACHARYA; RAO; TSAI, 1983).Assim, pela sua posição em energia, atribuímos tentativamente a origem do pico $\mathbf{P c}_{\mathbf{3}}$ posicionado em $0,797 \mathrm{eV}$ a uma transição envolvendo éxcitons. A largura de linha relativamente grande para uma transição excitônica está associada a flutuações de potencial causadas pelas variações aleatórias na composição das ligas ternárias (SCHUBERT; TSANG, 1986; BIMBERG; SROCKA, 1993; CHARREAUX; GUILLOT; NOUAILHAT, 1986).

O pico em nossa amostra está: $4 \mathrm{meV}$ abaixo da transição excitônica observada por Bassignana, Miner e Puetz (1989). De acordo com Bimberg e Srocka (1993), uma pequena variação na composição menor que $0.1 \%$ é suficiente para que ocorra uma mudança no "bandgap" de $1 \mathrm{meV}$. Além disso, a presença de flutuações de potencial implica na existência de estados de energia abaixo do "bandgap" que contribuem para a redução da energia de recombinação da transição excitônica, especialmente quando os espectros são obtidos em condições de baixa temperatura e baixa intensidade de excitação.
Portanto, esses dois efeitos podem estar contribuindo para esta diferença observada na energia de emissão da transição excitônica em nosso trabalho quando comparada à energia observada por Bassignana, Miner e Puetz (1989).

\section{Picos de Menor Energia $\mathrm{Pc}_{1}$ e $\mathrm{Pc}_{2}$}

Da Tabela IV, verificamos que as energias dos outros dois picos existentes em nossa amostra, à temperatura de 8K, correspondem a: $\mathbf{P c _ { 1 }}=0,772 \mathrm{eV}$ e $\mathbf{P c}_{2}=0,782 \mathrm{eV}$.

$\mathrm{O}$ pico $\mathbf{P c}_{\mathbf{2}}$ observado em $0,782 \mathrm{eV}$, corresponde a uma diferença de energia em relação à transição excitônica de $15 \mathrm{meV}$, enquanto o pico de energia menor $\mathbf{P c}_{\mathbf{1}}=0,772 \mathrm{eV}$, corresponde a uma diferença de energia, em relação à transição excitônica de $25 \mathrm{meV}$. Assim, pela posição em energia dos picos existentes em nossa amostra e pela comparação com os resultados apresentados na sessão de revisão da literatura (secção 2), é possível, a principio, imaginar tratar-se de emissões com participação de impurezas, provavelmente transições D-A com dois aceitadores diferentes.

Para o pico $\mathbf{P} \mathbf{c}_{2}$, algumas impurezas aceitadoras possíveis seriam o $\mathrm{Mg}$ (LOUATI et al., 1987), o Cd (CHEN et al., 1989), o C (CHARREAUX;GUILLOT; NOUAILHAT, 1986; YU; PENG; MORKOC, 1989) ou ainda o Zn (GOETZ et al., 1983; PEARSALL, EAVES; PORTAL, 1983; CHARREAUX; GUILLOT; NOUILHAT, 1986; DHAR et al., 1997). Estes dois últimos são os mais prováveis, já que o Cé tido como a impureza dominante em camadas de GaInAs crescidas por MBE (YU; PENG; MORKOC, 1989) e o Zn é relatado como uma impureza residual significante no In refinado (PEARSALL, EAVES; PORTAL, 1983).

Quanto ao pico de energia menor, $\mathbf{P c}_{1}$, as principais impurezas aceitadoras possíveis seriam o Be (FENG et al., 1979) e o Si (GOETZ et al., 1983; BHATTACHARYA et al., 1983).

Para confirmar quais os processos envolvidos nos picos de menor energia nos espectros de PL em nossa amostra, faremos uma análise das medidas de PL em 
função da temperatura e potência do laser de excitação.

\section{Fotoluminescência com Variação de Temperatura}

$\mathrm{Na}$ Figura 4, apresentamos os espectros de PL da amostra SR295 na região de emissão do InGaAs, onde variamos a temperatura entre 8 e $45 \mathrm{~K}$.

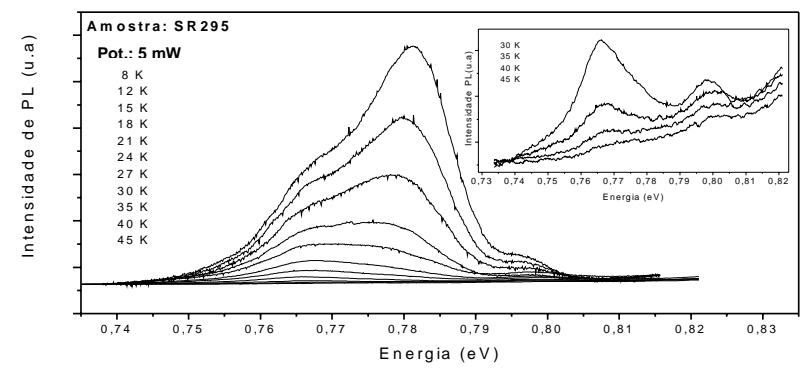

Figura 4. Espectro de fotoluminescência com variação de temperatura entre 8 e $45 \mathrm{~K}$ na região de emissão da camada "buffer" de InGaAs. A disposição dos espectros na figura obedece à ordem decrescente em relação à temperatura (seguindo a legenda apresentada no lado esquerdo da figura). A inserção apresenta uma ampliação dos espectros obtidos no intervalo de temperatura entre $30 \mathrm{~K} \mathrm{e} 45 \mathrm{~K}$.

Os espectros da Fig. 4 foram obtidos com baixas intensidades de excitação, condição que favorece a análise do comportamento das transições envolvendo impurezas. Por exemplo, a luminescência da emissão da banda de condução para o nível aceitador (e-A) deve ser realizada com intensidades de excitação especiais e em temperaturas apropriadas, de maneira que favoreça esta emissão em relação à emissão do par doador-aceitador (D-A). Geralmente, em amostras com dopagens baixas ou moderadas e em baixas temperaturas, a banda (D-A) domina o espectro quando tomadas em baixas intensidades de excitação.

Podemos observar claramente, na Fig. 4, a existência de pelo menos três canais de recombinação à baixas temperaturas. Também observamos que ocorrem algumas mudanças significativas nos picos à medida que a temperatura aumenta, como, por exemplo, o aparente deslocamento dos picos de energias mais baixas $\left(\mathbf{P c}_{\mathbf{1}}\right.$ e $\left.\mathbf{P} \mathbf{c}_{\mathbf{2}}\right)$ para menores energias com a temperatura, comportamento característico do "bandgap" dos materiais III-V. Podemos notar que todas as transições praticamente se extinguem para temperaturas em torno ou acima de $45 \mathrm{~K}$. Outra importante característica é que, quando a temperatura aumenta, o pico de energia intermediária $\left(\mathbf{P c}_{2}\right)$ torna-se menos intenso mais rapidamente que o pico de energia menor $\left(\mathbf{P c}_{1}\right)$, que passa a dominar o espectro para temperaturas em torno de $\sim 25 \mathrm{~K}$. Este comportamento é um sinal característico de que existem diferentes canais de recombinação (devido a impurezas ou defeitos) competindo em nossa amostra. Além disso, o pico de energia menor $\left(\mathbf{P c}_{1}\right)$, persiste até uma temperatura um pouco maior do que $\mathbf{P c}_{2}$.

$\mathrm{O}$ pico de energia maior $\mathbf{P c}_{3}$, embora seja menos intenso do que os outros, permanece até a temperatura de $\sim 45 \mathrm{~K}$ e diminui de intensidade com a temperatura muito menos acentuadamente do que os outros picos. Este comportamento reforça a identificação deste pico, como sendo devido à transição excitônica, considerando-se que, para esta transição, a densidade de estados é relativamente grande quando comparada à densidade de estados finita de transições envolvendo impurezas.

Outro fato interessante é que, com o aumento da temperatura, ocorre um alargamento do espectro e um ligeiro deslocamento para valores maiores ("blueshift") na energia da emissão excitônica. Isso pode ser observado na inserção da Fig. 4, na qual é apresentada uma ampliação dos espectros de PL para as temperaturas entre 30 e $45 \mathrm{~K}$. Estes efeitos podem ser explicados pela presença de flutuações no potencial de confinamento dos éxcitons. Como mencionamos anteriormente, em ligas ternárias como o InGaAs, a distribuição aleatória na composição provoca flutuações estatísticas de potencial que afetam as propriedades ópticas destas ligas. A interação dos éxcitons com estas flutuações de potencial provoca a formação de um "band-tail" na densidade de estados excitônica. Quando uma liga 
que não apresenta um nível muito grande de flutuações é excitada em condições de baixa temperatura e baixa intensidade de excitação, os éxcitons podem relaxar através da interação com fônons para o mínimo de potencial antes de a recombinação radiativa ocorrer. Quando a temperatura aumenta, os éxcitons passam a popular estados de energias mais altas do "band tail", resultando em um deslocamento do pico de PL para energias maiores ('blueshift") e um alargamento da linha da emissão.

Para complementar a análise do comportamento das transições em função da temperatura, fizemos uma série de ajustes da forma de linha dos espectros para cada temperatura. Porém, como será descrito a seguir, estes ajustes devem apresentar um erro relativamente grande por dois principais motivos:

- Como as transições estão superpostas, os ajustes podem apresentar erros muito grandes.

- Quando a temperatura aumenta, ocorre uma inclinação da linha de base do espectro.

Para ilustrar isto, nós mostramos na Fig. 5 alguns espectros com os respectivos ajustes em algumas temperaturas diferentes.

Figura 5. Espectros de PL para várias temperaturas, mostrando os ajustes por gaussianas.
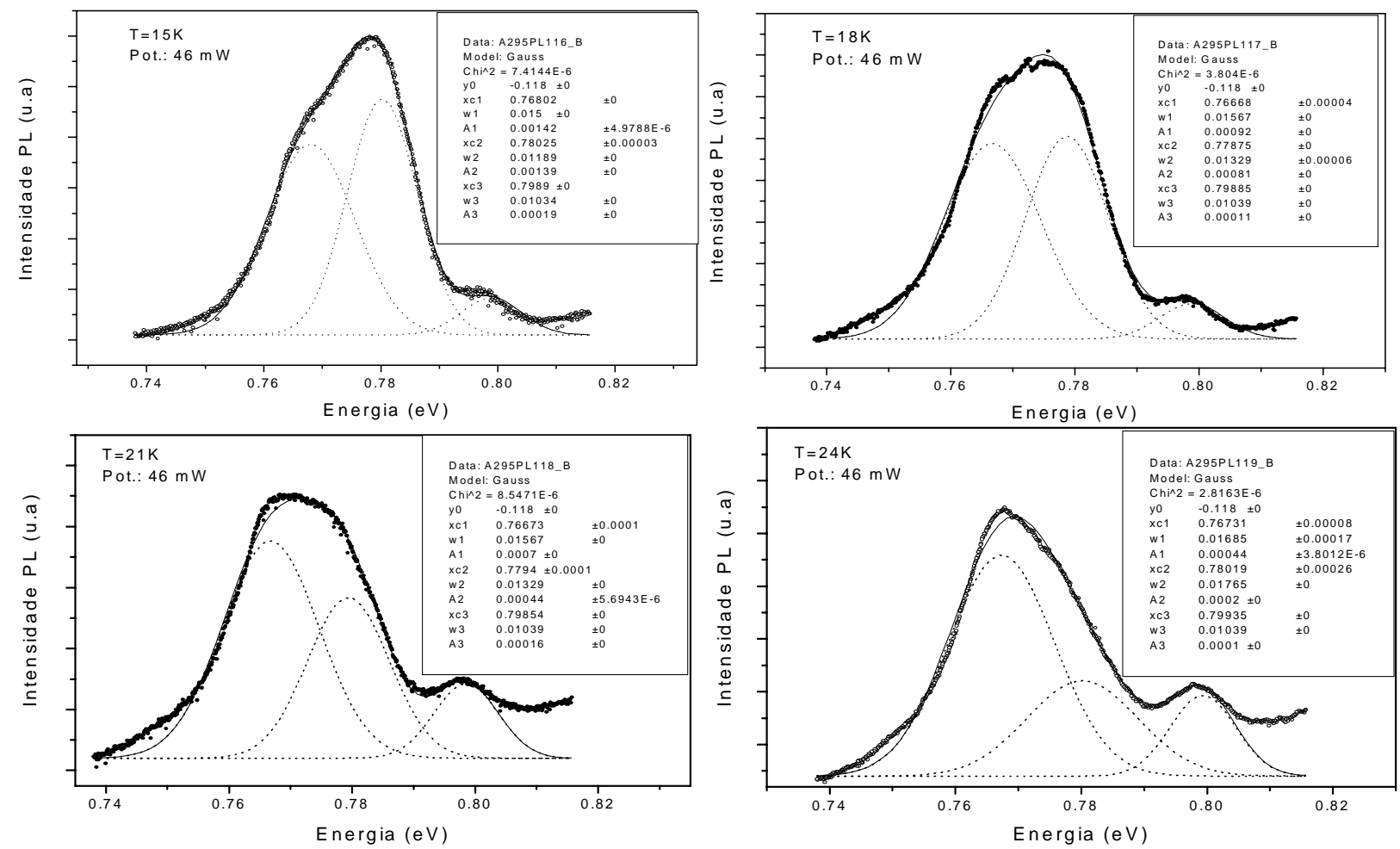

Podemos observar na Fig. 5, a inclinação da linha

Um dos objetivos de realizarmos os ajustes foi a de base dos espectros aumenta no lado de maior energia, à medida que a temperatura sobe. Esta inclinação é provocada por influência das transições relacionadas às duas super-redes existentes na amostra, que são extremamente mais intensas que a emissão da camada "buffer", como podemos observar na Fig. 2.

obtenção das intensidades integradas (área do pico) de cada emissão separadamente e, por meio de um gráfico do tipo "Arrenhius", estimar as energias de ativação dos diferentes processos envolvidos. Obtivemos, porém, energias de ativação muito baixas - especialmente para as transições de menor energia $\mathbf{P c}_{1}$ e $\mathbf{P c}_{2}$ - da ordem de 5-8 meV e atribuímos este fato às seguintes hipóteses: 
a) ocorre a imprecisão dos ajustes devido às dificuldades às quais já nos referimos,

b) considerando as transições observadas como recombinações entre pares D-A, as energias obtidas são iguais à energia necessária para popular os níveis doadores; assim, quando todos os níveis doadores estão ionizados, ter-se ia uma recombinação do tipo (e-A). Porém, o elétron foto-excitado para a banda de condução é capturado pelos defeitos existentes na amostra e recombina não radiativamente, provocando o desaparecimento da emissão.

c) no caso de transições envolvendo elétrons localizados nas regiões de flutuações de potencial da banda de condução e impurezas aceitadoras, o processo seria semelhante ao descrito em b) com a diferença de que, nesse caso, a energia obtida seria igual à magnitude das flutuações de potencial criadas pelas flutuações aleatórias da composição da liga. À medida que a temperatura aumenta, o elétron adquire energia térmica suficiente para escapar da flutuação de potencial em que está localizado e torna-se livre na banda de condução. Quando isto ocorre, o elétron pode ser capturado por defeitos e recombinar não radiativamente, provocando assim o desaparecimento da emissão.

\section{Fotoluminescência Com Variação da Potência de Excitação}

$\mathrm{Na}$ seqüência, vamos analisar as mudanças provocadas nos espectros de PL quando variamos a potência do laser de excitação. Na Fig. 6, apresentamos os espectros de PL obtidos à temperatura de $8 \mathrm{~K}$, onde variamos a potência do laser entre 0,25 e $100 \mathrm{~mW}$.

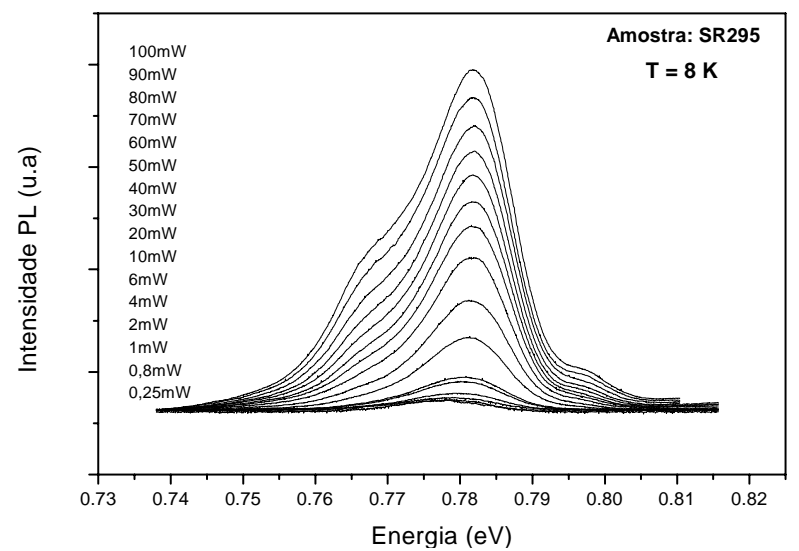

Figura 6. Espectros de fotoluminescência com variação de potência do laser de excitação entre $0,25 \mathrm{~mW}$ e 100 $\mathrm{mW}$ para a amostra SR295. A disposição dos espectros na figura obedece à ordem decrescente em relação à potência (seguindo a legenda apresentada no lado esquerdo da figura)

Analisando a Fig. 6, podemos observar um aumento na intensidade dos picos à medida que aumentamos a potência do laser de excitação e também um ligeiro deslocamento para energia maior do máximo do pico localizado em torno de $0.78 \mathrm{eV}$. Realizamos alguns ajustes da forma de linha para cada pico individualmente, afim de para obtermos a intensidade integrada da luminescência em função da potência do laser de excitação (veja a Fig. 7). Esses ajustes também são feitos para tentar identificar a origem deste deslocamento para energia maior - que poderia caracterizar a existência de transições entre pares D-A, uma vez que este é um comportamento característico deste processo. Porém, devido ao fato de que as transições $\mathbf{P c}_{\mathbf{1}}$ e $\mathbf{P} \mathbf{c}_{\mathbf{2}}$ estão superpostas, os ajustes não apresentaram dados suficientemente satisfatórios para que pudéssemos mensurar qual a contribuição individual de cada pico a este deslocamento. 


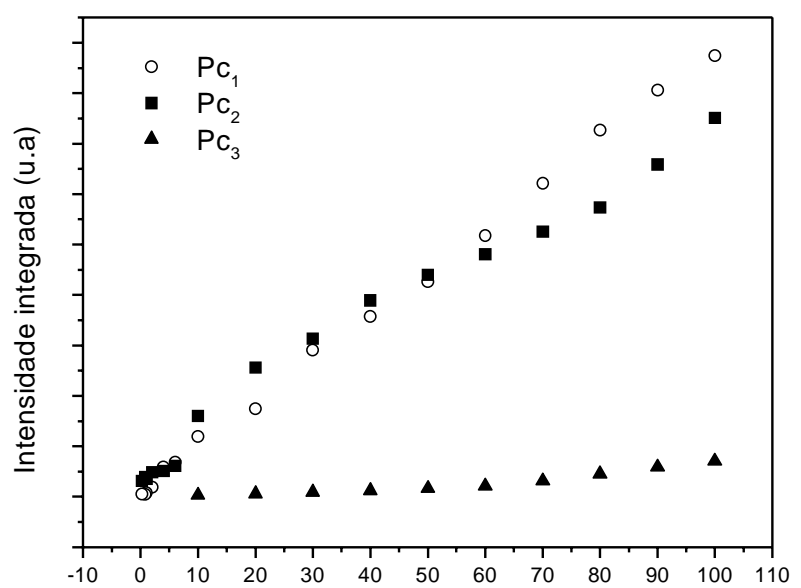

Figura 7. Intensidade Ratengeiad(n) função da potência do laser de excitação obtida dos ajustes dos espectros da Fig. 6.

$\mathrm{Na}$ literatura, temos que a intensidade integrada da luminescência varia com a potência de excitação de acordo com a relação: I oC $\mathrm{P}^{\mathbf{k}}$ (FERGUSSON et al., 1994; ZHONGYING et al., 1987; SCHMIDT; LISCHKA; ZULEHNER, 1992; ZHOU; TORRES, 1994; PAVESI; GUZZI, 1994; MOZUME et al.; 2000; FENG; MASCARENHAS; CHOYKE, 1986); onde I é a intensidade integrada da luminescência e $\mathbf{P}$ é a potência do laser de excitação. Assim, em um gráfico do tipo $\log \mathbf{I} x \log \mathbf{P}, \mathbf{k}$ representa a inclinação da curva dos pontos experimentais. Valores de $\mathbf{k}<1$ (comportamento sublinear) representam transições envolvendo impurezas e valores de $2>\mathbf{k}>1$ (comportamento superlinear) são obtidos quando se trata de transições envolvendo éxcitons (éxciton livre e ligado) (SCHMIDT; LISCHKA; ZULEHNER, 1992; PAVESI; GUZZI, 1994; FENG; MASCARENHAS; CHOYKE, 1986).

Na Figura 8, apresentamos os gráficos da intensidade integrada em função da potência em escala logarítmica para os espectros mostrados na Fig. 6. Os valores obtidos para as inclinações das curvas são mostrados na figura e serão transportados para a Tabela V. Para a transição $\mathbf{P} \mathbf{c}_{\mathbf{3}}$ foram obtidos dois valores de $\mathbf{k}$ quando as curvas são ajustadas considerando duas regiões de potências diferentes e um valor de k, quando foi considerada a inclinação para uma curva única.
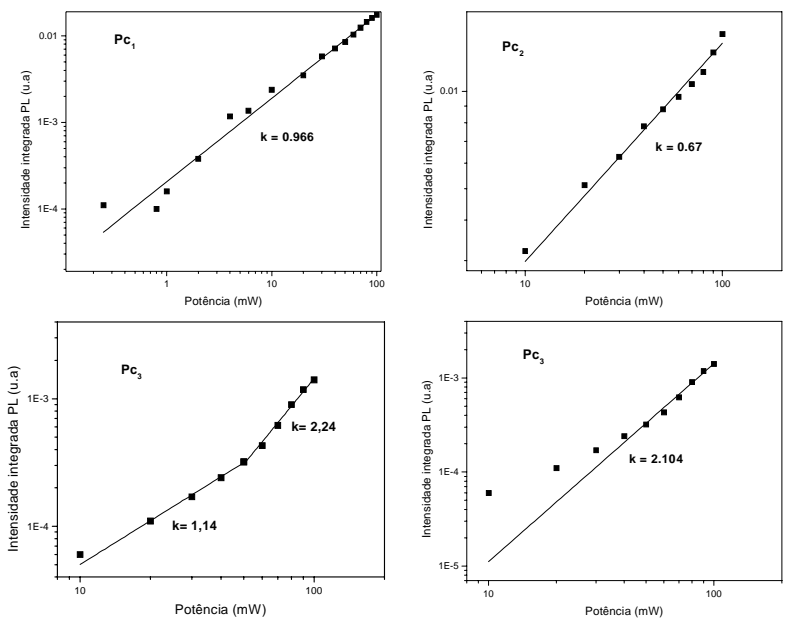

Figura 8. Intensidade integrada da luminescência em função potência de excitação.

Na Figura 9, apresentamos uma seqüência de medidas, na qual variamos a potência de excitação entre 0,08 a $150 \mathrm{~mW}$. Deve ser notado, porém, que devido às condições de alinhamento do sistema óptico, nessas medidas a intensidade de excitação é muito maior do que aquelas utilizadas na obtenção dos espectros da Fig. 6

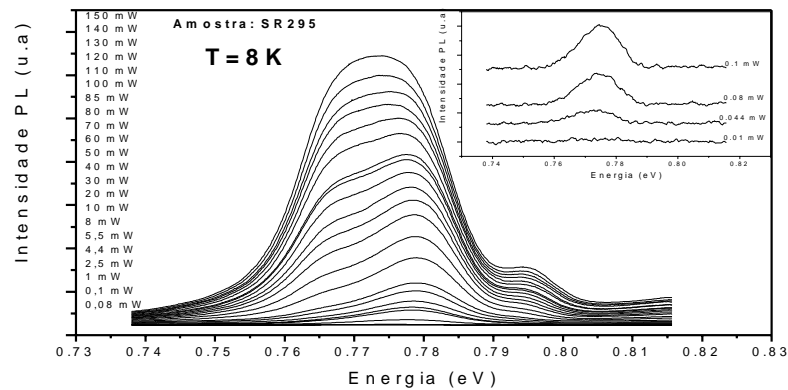

Figura 9. Espectros de PL com variação de potência entre 0,08 e $150 \mathrm{~mW}$ para a amostra SR295.

Analisando a Fig. 9, podemos observar que todas as transições aumentam de intensidade à medida que aumentamos a potência do laser de excitação. Porém, notamos claramente que a intensidade do pico de energia menor $\mathbf{P} \mathbf{c}_{1}$ aumenta muito mais do que a intensidade do pico de energia intermediária $\mathbf{P} \mathbf{c}_{2}$, que apresenta um comportamento de saturação, enquanto o pico $\mathbf{P} \mathbf{c}_{1}$ domina o espectro para potências maiores. Para as intensidades de excitação que utilizamos, o pico $\mathbf{P} \mathbf{c}_{1}$ não apresenta sinais de saturação, porém, 
acreditamos que isto deve ocorrer para intensidades de excitação maiores.

Realizamos para esta seqüência de medidas uma série de ajustes com gaussianas, da mesma forma como foi feito anteriormente, e apresentamos na Fig. 10 os gráficos da intensidade integrada em função da potência de excitação em escala logarítmica para os espectros mostrados na Fig. 9. Os valores obtidos para as inclinações $\mathbf{k}$ das curvas são mostrados na Fig. 10 e serão transportados para a Tabela V.

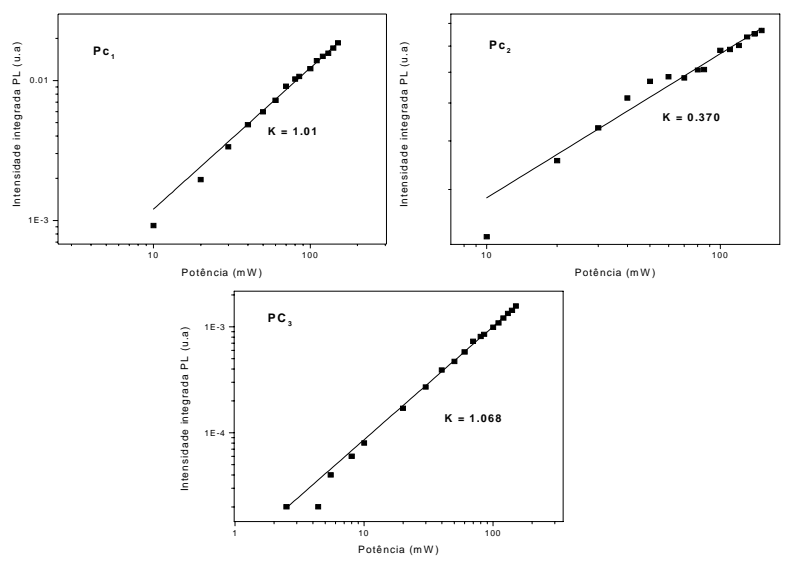

Figura 10. Gráfico da intensidade integrada da luminescência em função da potência de excitação.

Os valores obtidos para a inclinação $\mathbf{k}$ das curvas de intensidade integrada em função da potência de excitação para as duas sequiências de medidas de variação de potência (Figs. 6 e 9) são apresentados na Tabela V. Os valores de k obtidos da Fig. 8, considerando os espectros mostrados na Fig. 6, estão relacionados na tabela como "Medidas de PL baixa intensidade" enquanto que os valores obtidos para a inclinação retirados da Fig. 10, considerando os espectros da Fig. 9, estão relacionados na tabela na coluna "Medidas de PL alta intensidade".

Tabela V. Valores obtidos para a inclinação $\mathbf{k}$ da curva $\log$ I x $\log \mathrm{P}$.

\begin{tabular}{c|c|c|c}
\hline $\begin{array}{c}\text { Medidas de PL baixa } \\
\text { intensidade }\end{array}$ & $\mathbf{k}$ & $\begin{array}{c}\text { Medidas de PL } \\
\text { alta intensidade }\end{array}$ & $\mathrm{K}$ \\
\hline $\mathbf{P c}_{\mathbf{1}}$ & 0,96 & $\mathbf{P c} \mathbf{1}_{\mathbf{1}}$ & 1,01 \\
\hline $\mathbf{P c}_{\mathbf{2}}$ & 0,67 & $\mathbf{P c _ { 2 }}$ & 0,37 \\
\hline $\mathbf{P c}_{\mathbf{3}}$ & 2,1 & $\mathbf{P c _ { 3 }}$ & 1,06 \\
\hline $\mathbf{P c}_{\mathbf{3}}$ & $1,14 \mathrm{e} 2,24$ & - & - \\
\hline
\end{tabular}

Para analisarmos os dados da Tabela V, é preciso termos em mente que a obtenção dos valores para a intensidade integrada em cada potência para cada pico é feita através dos ajustes dos espectros e, como já citamos anteriormente, devido ao fato que as transições estão superpostas e que ocorre uma inclinação da linha de base do espectro (da mesma forma que ocorre com o aumento da temperatura ver, por exemplo, a Fig. 9), isso faz com que os ajustes dos picos fiquem assim pouco precisos, ou seja, pode haver um erro relativamente grande.

Como já citamos anteriormente, valores de $\mathbf{k}$ menores que a unidade correspondem a transições envolvendo impurezas. Este é, sem dúvida, o caso do pico de energia intermediária $\mathbf{P c}_{2}$, que nas duas seqüências de medidas apresenta valores de $\mathbf{k}$ bem menores que a unidade, o que assegura que esta emissão envolve impurezas. No caso do pico de energia menor $\mathbf{P} \mathbf{c}_{1}$, obtivemos para as medidas com intensidade baixas um valor ligeiramente abaixo da unidade, que aponta a presença de impurezas; porém, para a seqüência de medidas com intensidade de excitação maior, o valor de $\mathbf{k}$ obtido é ligeiramente maior do que a unidade. Levando em conta o fato de que deve haver um erro considerável na obtenção da intensidade integrada extraída dos ajustes, é bem provável que este pico também envolva impurezas, embora não possamos afirmar isso baseado apenas nesta análise. Quanto ao pico de energia maior $\mathbf{P c}_{3}$, nos espectros obtidos com potência mais baixa, este pico apresenta uma intensidade muito baixa, o que dificulta ainda mais a obtenção da intensidade integrada deste pico. Nós obtivemos valores de $\mathbf{k}>$ 2 quando foi considerada a inclinação para uma curva única e valores diferentes de $\mathbf{k}$ quando foi considerada a inclinação em duas regiões de potências diferentes: $\mathbf{k}=1,14$ para potências mais baixas e $\mathbf{k}=2,24$ para potências mais altas. $\mathrm{Na}$ seqüência de medidas com potência mais alta, que acreditamos apresentar uma melhor condição para obter a intensidade integrada deste pico devido ao fato que nestas medidas este apresenta uma intensidade maior sendo assim mais fácil de ajustar, nós encontramos um valor de $\mathbf{k}=1,06$, ou seja, um comportamento superlinear, o que comprova o caráter excitônico desta transição. 


\section{Discussão dos Resultados Experimentais}

Da análise das medidas de fotoluminescência obtidas com variação de temperatura e potência e pela comparação com os resultados publicados na literatura, nós atribuímos as origens dos picos de emissão observados, como segue:

$\mathrm{O}$ pico $\mathbf{P c}_{3}$ observado em nossa amostra à temperatura de $8 \mathrm{~K}$ em $0,797 \mathrm{eV}$ tem sua origem em transições envolvendo éxcitons localizados que ocorrem na camada "buffer" de InGaAs. Esta conclusão é baseada nos seguintes argumentos:

- Desaparecimento desta emissão com aumento da temperatura, mas com diminuição menos acentuada do que as demais emissões.

- Comportamento superlinear da intensidade integrada em função da potência de excitação.

- Posição espectral comparável aos valores atribuídos para esta transição na literatura.

A largura de linha relativamente grande para uma transição excitônica e o deslocamento do pico de PL para energia maior ("blueshift"), observado com aumento da temperatura, estão associados às flutuações de potencial causadas pela variação aleatória na composição das ligas ternárias (SCHUBERT; TSANG, 1986; BIMBERG; SROCKA, 1993; CHARREAUX; GUILLOT; NOUAILHAT, 1986).

O pico em nossa amostra está $4 \mathrm{meV}$ abaixo do observado por Bassignana, Miner e Puetz (1989) para a transição envolvendo éxciton ligado, fato devido a duas contribuições: a) as flutuações de potencial a que já nos referimos acima e que fazem com que hajam estados com energia abaixo da energia do éxciton livre (criando assim os éxcitons localizados) e b) uma pequena diferença na composição da liga, com relação à composição de rede casada.

Pela comparação com os resultados experimentais publicados na literatura (secção 2) e pelo comportamento da intensidade integrada da luminescência em função da potência de excitação (comportamento sublinear mostrado na Tabela V), atribuímos o pico de energia intermediária $\mathbf{P c}_{\mathbf{2}}$ à transições envolvendo impurezas aceitadoras.
Quanto ao pico de energia menor $\mathbf{P} \mathbf{c}_{\mathbf{1}}$, verifica-se que o comportamento quase linear da intensidade integrada da luminescência em função da potência de excitação (Tabela $\mathrm{V}$ ), pode representar um processo no qual não haja a participação de impurezas. Devido à sua posição espectral e comportamento com a temperatura, também atribuímos esse pico à transições envolvendo impurezas aceitadoras, porém com energia de ligação maior do que a energia de ligação das impurezas envolvidas em $\mathbf{P c}_{2}$.

Devido ao fato de que as transições estão superpostas, a análise das medidas de PL com variação de temperatura e potência não forneceram dados suficientemente conclusivos que permitissem determinar inequivocamente se os processos envolvidos em $\mathbf{P} \mathbf{c}_{2}$ e $\mathbf{P} \mathbf{c}_{1}$, estão relacionados a transições da banda de condução para nível aceitador (e-A) ou transições entre o par doador-aceitador (D-A). No entanto, o fato das emissões se extinguirem com o aumento da temperatura, a temperaturas baixas, faz-nos crer, como discutido anteriormente, que o processo envolvido nos dois casos seja a transição D-A e/ou uma transição envolvendo elétrons localizados na região de flutuação de potencial da banda de condução e impurezas aceitadoras $\left(\mathrm{e}_{\mathrm{loc}}-\mathrm{A}\right)$.

Goetz et al. (1983) observaram que as energias de ligação dos aceitadores no InGaAs eram em torno de $5 \mathrm{meV}$ maiores do que a separação em energia entre o pico do éxciton e a transição D-A. O pico $\mathbf{P c}_{2}$ está posicionado $15 \mathrm{meV}$ abaixo da transição excitônica; isto corresponderia, no caso de uma transição D-A, a uma impureza aceitadora com energia de ionização de $\sim 20 \mathrm{meV}$. As principais impurezas candidatas seriam $\mathrm{Mg}, \mathrm{Cd}, \mathrm{C}$ ou $\mathrm{Zn}$.

$\mathrm{O}$ pico Pc $\mathbf{P c}_{1}$ está posicionado $25 \mathrm{meV}$ abaixo da transição excitônica, o que corresponderia, no caso de uma transição D-A, a uma impureza aceitadora com uma energia de ionização maior, em torno de $30 \mathrm{meV}$. Neste caso, os principais candidato seriam o Be e o $\mathrm{Si}$.

\section{Conclusões}

Analisando os resultados das medidas de fotoluminescência em função da temperatura e da potência de excitação e comparando as energias das 
emissões observadas a baixas temperaturas com os resultados publicados na literatura, atribuímos as origens dos três picos de emissão observados na camada "buffer" de InGaAs como segue:

a) o pico de emissão observado em $0,797 \mathrm{eV}$ a $8 \mathrm{~K}$ foi atribuído a transições envolvendo éxcitons localizados em regiões de flutuação de potencial;

b) os outros dois picos de emissão, localizados em $0,772 \mathrm{eV}$ e $0,782 \mathrm{eV}$, foram atribuídos a transições envolvendo impurezas aceitadoras.

Devido à superposição das transições de energia mais baixa, a análise dos espectros de PL não forneceu dados suficientemente conclusivos que permitissem determinar inequivocamente se os processos envolvidos nestas transições estão relacionados a transições da banda de condução para o nível aceitador (e-A) ou transições entre o par doador-aceitador (D-A). No entanto, devido ao comportamento dessas transições com o aumento da temperatura, acreditamos que o processo envolvido nos dois casos seja a transição D-A e/ou uma transição envolvendo elétrons localizados na região de flutuação de potencial da banda de condução e impurezas aceitadoras $\left(\mathrm{e}_{\mathrm{loc}}-\mathrm{A}\right)$.

\section{Agradecimentos}

Agradecemos à CAPES, CNPq, FBB e Fundação Araucária pelo apoio financeiro.

\section{Referências}

BASSIGNANA, I. C.; MINER, C. J.; PUETZ, N. Photoluminescence and double-crystal x-ray study of InGaAs/InP: Effect of mismatch strain on band gap. Journal of Applied Physics, New York, v.65, p.4299, 1989.

BHATTACHARYA, P. K.; RAO, M. V.; TSAI, M. Growth and photoluminescence spectra of high-purity liquid phase epitaxial $\mathrm{In}_{0.53} \mathrm{Ga}_{0.47}$ As. Journal of Applied Physics, New York, v.54, p.5096, 1983.

BIMBERG, D.; SROCKA, B. Datareview: photoluminescence of pure InGaAs alloys - Properties of lattice-matched and strained Indium Gallium Arsenide. London: University of Michigan, 1993. p.159-168.

BIMBERG, D.; SROCKA, B. Datareview: Impurity level in InGaAs - Properties of lattice-matched and strained Indium Gallium Arsenide. London: University of Michigan, 1993. p.169-173.
BÖHRER, J.; KROST, A.; BIMBERG, D. B. Composition dependence of band gap and type of lineup in $\mathrm{In}_{1-\mathrm{x}-\mathrm{y}} \mathrm{Ga}_{\mathrm{x}} \mathrm{Al}_{\mathrm{y}} \mathrm{As} / \mathrm{InP}$ heterostructures. Applied Physics Letters, New York, v.63, p.1918, 1993.

CHARREAUX, C.; GUILLOT, G.; NOUAILHAT, A. Alloy broadening in photoluminescence spectra of $\mathrm{Ga}_{0.47} \mathrm{In}_{0.53}$ As. Journal of Applied Physics, New York, v.60, p.768, 1986.

CHEN, Y.; KIM, O. K.; Near-band gap absorption and photoluminescence of $\mathrm{In}_{0.53} \mathrm{Ga}_{0.47}$ As semiconductor alloy. Journal of Applied Physics, New York, v.52, p.7392, 1981.

CHEN, Z.; KORB, W.; BAUER, R. K.; BIMBERG, D.; First observation of a titanium midgap donor level in $\mathrm{In}_{0.53} \mathrm{Ga}_{0.47}$ As $p$ - $n$ diodes. Applied Physics Letters, New York, v.55, p.645, 1989.

DAI, Y. T.; FAN, J. C.; CHEN, Y. F.; Studies of twosubband occupied electron gas in modulation-doped $\mathrm{In}_{0.52} \mathrm{Al}_{0.48} \mathrm{As} / \mathrm{In}_{0.53} \mathrm{Ga}_{0.47} \mathrm{As}$ single quantum well by farinfrared modulated photoluminescence. Journal of Applied Physics, New York, v.83, p.2127, 1998.

DHAR, S.; PAUL, S.; MAZUMDAR, M.; BANERJEE, S.; Effect of Er dopant on the properties of $\operatorname{In}_{0.53} \mathrm{Ga}_{0.47} \mathrm{As}$ layers grown by liquid phase epitaxy. Journal of Applied Physics, New York, v.81, p.2391, 1997.

DORREN, B. H. P.; SILOV, A. Y.; LEYS, M. R.; HAVERKORT, J. E. M.; WOLTER, J. H.; Electrorefraction in strained InGaAs/InP chopped quantum wells: Significance of the interface layers. Journal of Applied Physics, New York, v.87, p.2331, 2000

FENG, M.; OBERSTAR, J. D.; WINDHORN, T. H.; COOK, L. W.; STILLMAN, G. E.; STREETMAN, B. G. Be-implanted 1.3- $\mu \mathrm{m} \mathrm{InGaAsP}$ avalanche photodetectors. Applied Physics Letters, New York, v.34, p.591, 1979.

FENG, Z. C.; MASCARENHAS, A.; CHOYKE, W. J. Low temperature photoluminescence spectra of (001) CdTe films grown by molecular beam epitaxy at different substrate temperatures. Journal of Luminescence, Amsterdam, v.35, p.329, 1986.

FRY, K. L.; KUO, C. P.; COHEN, R. M.; STRINGFELLOW, G. B.; Photoluminescence of organometallic vapor phase epitaxial GaInAs. Applied Physics Letters, New York, v.46, p.955, 1985.

GASKILL, D. K.; BOTTKA, N.; AINA, L.; MATTINGLY, M.; Band-gap determination by photoreflectance of InGaAs and InAlAs lattice matched to InP. Applied Physics Letters, New York, v.56, p.1269, 1990.

GEDDO, M.; BELLANI, V.; GUIZZETTI, G.; Optical study of the strain effect in pseudomorphic $\mathrm{In}_{1-x} \mathrm{Ga}_{x} \mathrm{As}$ InP heterostructures. Physical Review B, New York, v.50, p.5456, 1994.

GINIGE, R.; CHERKAOUI, K.; WONG KWAN, V.; KELLEHER, C.; CORBETT, B.; High injection and carrier pile-up in lattice matched InGaAs/InP PN diodes for thermophotovoltaic applications, Journal of Applied Physics, New York, v.95, p.2809, 2004. 
GOETZ, K. H.; BIMBERG, D.; JÜRGENSEN, H.; SELDERS, J.; SOLOMONOV, A. V.; GLINSKII, G. F.; RAZEGHI, M.; Optical and crystallographic properties and impurity incorporation of $\mathrm{Ga}_{x} \mathrm{In}_{1-x}$ As $(0.44<x<0.49)$ grown by liquid phase epitaxy, vapor phase epitaxy, and metal organic chemical vapor deposition. Journal of Applied Physics, New York, v.54, p.4543, 1983.

HYBERTSEN, M. S.; Band offset transitivity at the InGaAs/InAlAs/InP(001) heterointerfaces. Applied Physics Letters, New York, v.58, p.1759, 1991.

LEWÉN, R.; MAXIMOV, I.; SHORUBALKO, I.; SAMUELSON, L.; THYLÉN, L.; XU, H. Q.; High frequency characterization of a GaInAs/InP electronic waveguide T-branch switch. Journal of Applied Physics, New York, v.91, p.2398, 2002.

LOUATI, A.; CHARREAUX, C.; A.NOUAILHAT, A.; GUILLOT, G.; FAVENNEC, P. N.; SALVI, M.; Photoluminescence studies of $\mathrm{Mg}$ and $\mathrm{Hg}$ implanted $\mathrm{Ga}_{0.47} \mathrm{In}_{0.53}$ As. Solid State Communications, New York, v.62, p.31, 1987.

LUGAND, C.; BENYATTOU, T.; GUILLOT, G.; VENET, T.; GENDRY, M.; HOLLINGER, G.; SERMAGE, B.; Type II recombination and band offset determination in a tensile strained InGaAs quantum well. Applied Physics Letters, New York, v.70, p.3257, 1997.

MORRAL, A. F.; ZAHLER, J. M.; ATWATER, H. A.; AHRENKIEL, S. P.; WANLASS, M. W.; InGaAs/InP double heterostructures on InP/Si templates fabricated by wafer bonding and hydrogen-induced exfoliation. Applied Physics Letters, New York, v.83, p.5413, 2003.

MOZUME, T.; GEORGIEV, N.; NISHIMURA, T.; YOSHIDA, H.; NISHIKAWA, S.; NEOGI, A.; Photoluminescence characterization of type II InGaAs/ $\mathrm{AlAsSb}$ heterostructures lattice matched to InP grown by molecular beam epitaxy. Journal of Crystal Growth, Amsterdam, v.209, p.445, 2000.

PAVESI, L.; GUZZI, M.; Photoluminescence of $\mathrm{Al} \mathrm{Ga}$ As alloys. Journal of Applied Physics, New York, v.75, p.4779, 1994.

PEARSALL, T. P.; Datareview: InGaAs photodetectors: properties of lattice-matched and strained indium Gallium Arsenide. London: University of Michigan, 1993. p.267-278.

PEARSALL, T. P.; EAVES, L.; PORTAL, J. C. Photoluminescence and impurity concentration in $\mathrm{Ga}_{\mathrm{In}}$ ${ }_{x}$ As $\mathrm{P}_{1-y}$ alloys lattice-matched to InP. Journal of Applied Physics, New York, v.54, p.1037, 1983.

RADHAKRISHNAN, K.; PATRICK, T. H. K.; ZHENG, H. Q.; ZHANG, P. H.; YOON, S. F. Optical characterisation on the effect of doping concentration in InGaAs/InP HEMT structures. Microelectronic Engineering, Amsterdam, v.51-52, p .441, 2000.

SCHMIDT, T.; LISCHKA, K.; ZULEHNER, W. Excitation-power dependence of the near-band-edge photoluminescence of semiconductors. Physical Review B, New York, v.45, p.8989, 1992.
SCHUBERT, E. F.; TSANG, W. T.; Photoluminescence line shape of excitons in alloy semiconductors. Physical Review B, New York, v.34, p.2991, 1986.

SENGUPTA, D. K.; JACKSON, S. L.; CURTIS, A.. P.; FANG, W.; MALIN, J. I.; HORTON, T. U.; KUO, H. C.; MOY, A.; MILLER, J.; HSIEH, K. C.; CHENG, K. Y.; CHEN, H.; ADESIDA, I.; CHUANG, S. L.; FENG, M.; STILLMAN, G. E.; WU, W.; TUCKER, J.; CHANG, Y. C.; LI, L.; LIU, H. C. Growth and Characterization of InGaAs/InP p-Quantum-Well Infrared Photodetectors with Extremely Thin Quantum Wells. Journal of Electronic Materials, Warrendale, v.26, p.1382, 1997.

SWAMINATHAN, V.; STALL, R. A.; MACRANDER, A. T.; WUNDER, R. J.; Photoluminescence characterization of molecular beam epitaxy grown $\mathrm{In}_{x} \mathrm{Ga}_{1}$ As $(0.51<x<0.57)$. Journal Of Vacuum Science And Technology B, New York, v.3, p.1631, 1985.

TOWE, E.; Photoluminescence of undoped $\mathrm{In}_{0.53} \mathrm{Ga}_{0.47} \mathrm{As} /$ InP grown by the vapor phase epitaxy technique, Journal of Applied Physics, New York, v.53, p.5136, 1982

TROCCOLI, M..; CAPASSO, F.; CHEN, J.; PEABODY, M. L.; GMACHL, C.; SIVCO, D. L.; CHEN, C. H., CHO, A. Y.; Midinfrared electroluminescence in quantum cascade structures with $\mathrm{InP} / \mathrm{InGaAs}$ active regions; Journal of Applied Physics, New York, v.94, p.7101, 2003.

ZHONGYING, X.; JIZONG, X.; WEIKUN, G.; BAOZHEN, Z.; JUNYING, X.; YUZHANG, L.; The excitonic properties and temperature behaviour of the photoluminescence from GaAs-GaAlAs multiple quantum well structures. Solid State Communications, New York, v.61, p.707, 1987.

ZHOU, H. P.; TORRES, C. M. S.; Low-temperature emission of $\mathrm{Al}_{048} \mathrm{In}_{0.52} \mathrm{As}$ under high pressures, Journal of Applied Physics, New York, v.75, p.3571, 1994.

WEISS, B. L.; CHAN, Y.; SHIU, W. C.; LI, E. H.; The electro-optic properties of interdiffused InGaAs/InP quantum well structures; Journal of Applied Physics, New York, v.88, p.3418, 2000.

WEHMANN, H. H.; FIEDLER, F.; SCHLACHETZKI, A.; Activation energy of $\mathrm{Cd}$ in $\mathrm{In}_{1-\mathrm{x}} \mathrm{Ga}_{\mathrm{x}} \mathrm{As}_{\mathrm{y}} \mathrm{P}_{1-\mathrm{y}}$ on InP. Electronics Letters, London, v.22, p.1338, 1986 .

YANG, Q. K.; CHEN, J. X.; LI, A. Z.; Growth and characterization of high-quality GaInAs/AlInAs triple wells. Journal of Crystal Growth, Amsterdam, v.209, p.8, 2000.

YU, P. W.; KUPHAL, E.; Photoluminescence of Mn- and Un-doped $\mathrm{Ga}_{0.47} \mathrm{In}_{0.53} \mathrm{As}$ on InP. Solid State Communications, New York, v.49, p.907, 1984.

YU, P. W.; PENG, C. K.; MORKOÇ, H. Quasi-donoracceptor pair photoluminescence emission in $\mathrm{Ga}$ In $\mathrm{As} / \mathrm{InP}$. Journal of Applied Physics, New York, v.65, p.2427, 1989. 